\title{
Rotational Evolution of Intermediate and Low Mass Main Sequence Stars
}

\author{
John R. Stauffer \\ SIRTF Science Center, Keith-Spalding Building, MS 314-6, Caltech, \\ Pasadena, CA 91125
}

\begin{abstract}
Bob Kraft (1967) showed that there is a break in the mean rotational velocity of stars at about spectral type F5, with more massive stars generally being rapid rotators and less massive stars generally being slow rotators. He also showed that in the late $\mathrm{F}$ spectral range at least, there is an evolution with time on the main sequence, with younger $F$ stars being more rapidly rotating. Kraft's observational database extended only to about one solar mass due to the sensitivy limitations of photographic plates. Modern observations of low mass stars in open clusters, extending down in mass to nearly the hydrogen burning mass limit in a few clusters, have since been used to show that rotational spindown is the common feature of stars less massive than the sun but that there is a wide spread in rotational velocities when stars arrive on the ZAMS. I will review what is known empirically concerning the rotational velocities of intermediate and low mass field stars, using the open cluster data to place the field star observations in context.
\end{abstract}

\section{Introduction}

My historical context for the subject of this meeting took place during my first year in graduate school. At that time, U.C. Berkeley required that their astronomy graduate students demonstrate the ability to understand papers written in at least one other language where a significant set of published papers existed. To fulfill that requirement, incoming students needed to translate an article in the language of their choice into English. The article I chose to fulfill that requirement was Schatzman (1962), which had been published in French. That paper provided, I believe, the first quantitative model for rotational spindown of low mass, main sequence stars - and provided me an introduction to a topic which has interested me and in which I have worked ever since.

Because in principle we know the ages of the stars in open clusters, those clusters provide the best empirical data from which to determine how the rotational velocities of main sequence stars evolve with time. For that reason, I will spend most of my time reviewing what has been learned during the past two decades concerning stellar rotation in open clusters. In order to make even that task feasible, I will primarily concentrate on the two best-observed clusters - the Pleiades and the Hyades. I will briefly mention rotational data for a few other clusters, mostly in order to illustrate that their data are consistent with the evolutionary trend shown by comparison of the older Hyades cluster to the 
Pleiades. Similarly, I will only briefly review field star rotational velocities, in that case primarily to illustrate that those data are consistent with what is seen in the open clusters. In order to connect my talk to the theoretical talks later in the meeting, I will outline the "standard model" and illustrate the specific features in the observational data which motivated the assumptions adopted by the standard model. Finally, I will describe a few correlations between rotation and other observed properties of low mass stars which provide clues to the physical processes involved in shaping the rotational velocity distributions seen in open clusters.

\section{The Rotational Velocity Distribution for Low Mass Stars in the Pleiades}

Rotational velocities are now known for low mass stars in a number of open clusters. By far the best studied of the lot, however, is the Pleiades. The Pleiades is the only open cluster for which there is well-sampled rotational velocity data over the whole range from 0.1 to 1.0 solar masses. In part, this is because the Pleiades is fairly nearby, rich and in the northern hemisphere. However, at an age of about $100 \mathrm{Myr}$, the Pleiades is also at an interesting astrophysical age. For the purposes of rotational evolution of low mass stars, the Pleiades can to a large extent be considered a "ZAMS" cluster. That is, the age of the Pleiades is essentially equal to the PMS contraction age of stars with mass about $0.7 \mathrm{M}_{\odot}-$ so the Pleiades stars of that mass directly indicate the rotational distribution that those stars have upon arrival on the main sequence (and prior to any main sequence angular momentum loss). The Pleiades G stars arrived on the main sequence earlier, and so have undergone some spindown but probably not a lot. Similarly, the $\mathrm{M}$ dwarf Pleiades members are still contracting to the main sequence, but by $100 \mathrm{Myr}$ they do not have much spinup to go and so their rotational distribution is also not far from what it will be on the ZAMS. So, if one wanted to study a single cluster and use it to infer the ZAMS distribution of rotation for low mass stars, the Pleiades is as good a choice as any, and better than most.

Nearly 300 low mass Pleiades members now have published spectroscopic rotational velocities (Jones, Fischer \& Stauffer 1996; Oppenheimer et al. 1997; Queloz et al. 1998; Terndrup et al. 2000). Perhaps as important, all but a handful of those stars have measured rotational velocities as opposed to upper limits. Figure 1a shows a representation of the distribution of rotational velocities for low mass stars in the Pleiades. What I believe is important to remember from this figure are:

* there is a large spread in rotation over the entire mass range;

* the fastest rotators have $\mathrm{v}(\mathrm{rot}) \sim 1 / 3$ breakup, or periods of order 5 hours;

* the slowest rotators are remarkably slow, with $v \sin i \sim 5 \mathrm{~km} \mathrm{~s}^{-1}$;

* there appears to be a bimodal distribution of rotation for 0.6 to 0.9 solar masses, with well-defined loci of very slow rotators and very fast rotators; and * there appears to be a decreased range in rotation for the latest M dwarfs for which we have data. 

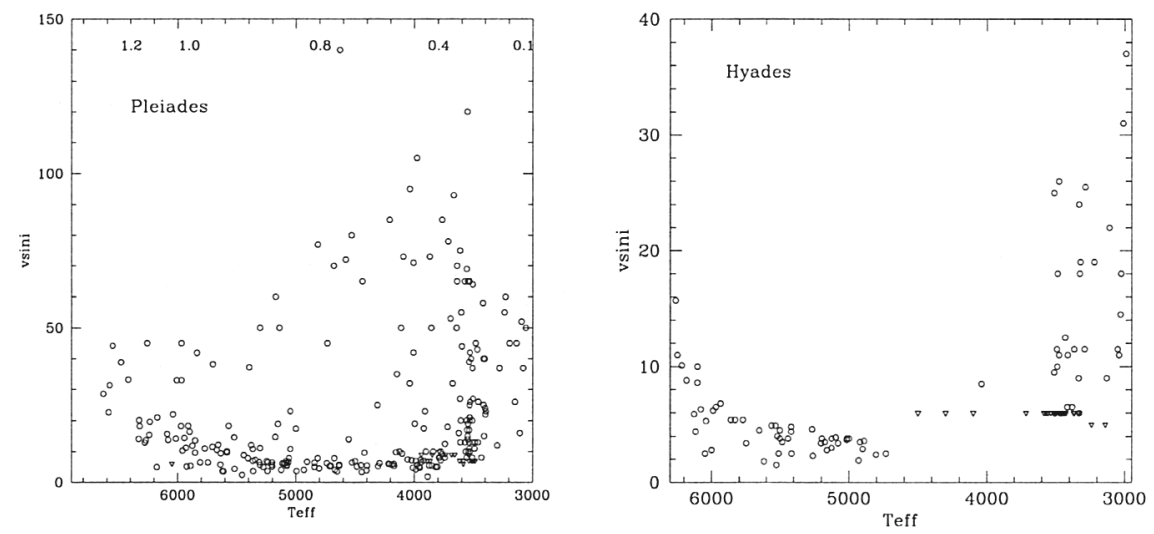

Figure 1. Rotational velocity distributions for the Pleiades (age $\sim 100 \mathrm{Myr}$ ) and Hyades (age $\sim 600 \mathrm{Myr}$ ). The scale at the top of the Pleiades figure provides approximate masses. Downward pointing triangles indicate stars for which only $v \sin i$ upper limits have been published. In neither cluster are there complete $v \sin i$ samples; we do not believe the stars observed are significantly biased to large or small rotation rates, however.

\section{The Rotational Velocity Distribution for Low Mass Stars in the Other Open Clusters}

The cluster with the next-best corresponding rotational velocity data is the Hyades. At an age of about $600 \mathrm{Myr}$, the Hyades is significantly older than the Pleiades, and thus one expects that main-sequence angular momentum loss will have modified the ZAMS distribution considerably. Figure $1 \mathrm{~b}$ shows the rotational velocity distribution for the Hyades (Jones, Fischer \& Stauffer 1996; Terndrup et al. 2000; Paulson \& Cochran 2002).

The salient features of this figure are:

* compared to the Pleiades, the Hyades low mass stars rotate systematically much more slowly, on average;

* the range in rotation at a given mass is also lower, in general; and

* the least spindown from Pleiades age occurs for the $\mathrm{M}$ dwarfs.

Figure 2 shows a comparison of the rotational velocity distributions of two additional clusters whose ages are intermediate between that of the Pleiades and Hyades. The dashed line in all four plots is a rough fit to the upper envelope of the Pleiades rapid rotators; the lower curve in all four plots is a rough fit to the Hyades distribution. The figure indicates that the low mass stars in the two intermediate age clusters have rotational velocities that are intermediate between that of the Pleiades and Hyades, in accord with the simple picture that, to first order, one can treat the rotational velocity distribution of each open cluster as a snapshot in time of the evolution of a single parent population. 


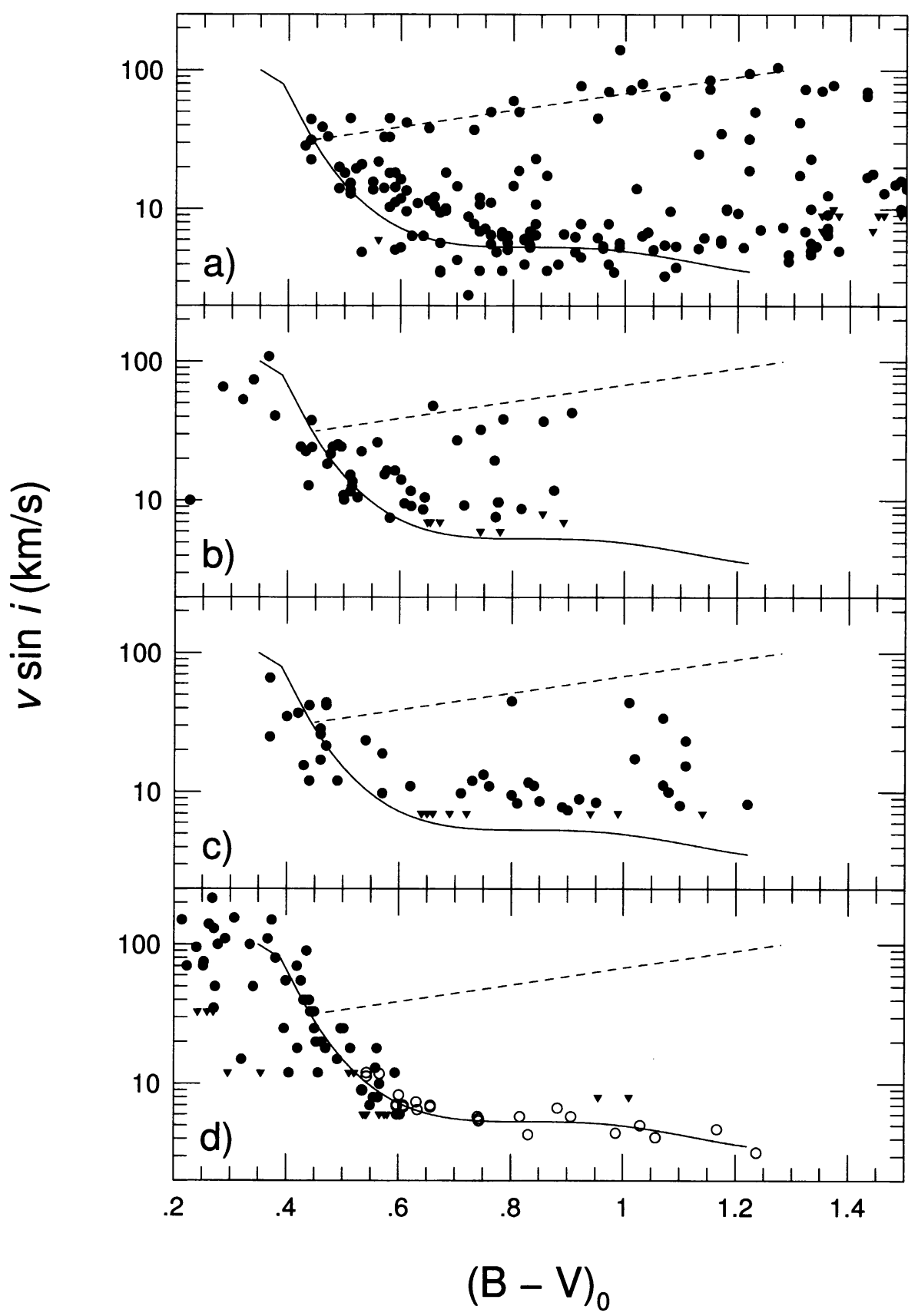

Figure 2. Rotational velocity distribution for four clusters - from top to bottom, Pleiades (age $100 \mathrm{Myr}$ ), NGC2516 (age $200 \mathrm{Myr}$ ), M34 (age $\sim 200 \mathrm{Myr}$ ) and Hyades (age 600 Myr). The NGC2516 $v \sin i$ 's are from Terndrup et al. 2002; the M34 $v \sin i$ 's are from Soderblom, Jones \& Fischer 2001. 


\section{The "Standard Model" for Rotational Velocity Evolution of Young, Low Mass Stars}

As indicated by Figures 1 and 2, and by the rotational velocity data available for pre-main sequence stars as discussed by Mathieu (2002), we now have a rich empirical database describing the rotational evolution of low mass stars. In order to attempt to provide a physical explanation for the empirical record, the theoretical models of this angular momentum evolution have become more complex over time. The current standard model (e.g. Queloz et al. 1998; Sills, Pinsonneault \& Terndrup 2000; Barnes, Sofia \& Pinsonneault 2001) includes the following primary components: "disk locking" during early PMS evolution; angular momentum loss via a scaled-up solar wind $\left(\mathrm{d} \omega / \mathrm{dt} \sim \omega^{3}\right.$, or $\left.\omega \propto \mathrm{t}^{-1 / 2}\right)$ for low and moderate rotational velocities; "saturation" of the angular momentum loss rate at high rotational velocities $\left(\mathrm{d} \omega / \mathrm{dt} \sim \omega\right.$, or $\left.\omega \propto \omega_{o} \mathrm{e}^{-\alpha t}\right)$, with the critical velocity above which the loss rate changes possibly being a function of mass; and core-envelope rotational decoupling during PMS and early mainsequence evolution.

Several speakers who follow me will discuss the theoretical models in detail. Therefore, I will only briefly indicate how each of these main features are motivated by the open cluster data. Disk locking - or, some other mechanism which provides an additional angular momentum loss during PMS evolution - is needed in order to explain the very slow rotation of the most slowly rotating stars seen in the Pleiades and in a few somewhat even younger clusters such as IC2391, IC2602 and Alpha Persei. Wind models do not predict enough angular momentum loss during the PMS epoch (by a large factor) to explain these stars, and if they did, they would then predict much too fast main-sequence spindown at later ages. The general decrease in rotation rate with age as indicated in Figure 2 provides evidence for continuous angular momentum loss on the main sequence, most easily ascribed to a wind. A solar-wind loss law $\left(\mathrm{d} \omega / \mathrm{dt} \sim \omega^{3}\right)$ naturally explains the decrease in the range in rotation with time for the $\mathrm{G}$ and $\mathrm{K}$ dwarfs. Saturation of the angular momentum loss rate at high rotational velocities was suggested by analogy with the dependence of chromospheric and coronal fluxes on rotation, as illustrated in Figure 3a. The fact that the range in rotation for the late $\mathrm{M}$ dwarfs does not decrease significantly between Pleiades and Hyades age suggests that most of those stars have rotational velocities above the critical saturation velocity (for that mass) for most of this age range (Terndrup et al. 2000). Finally, core-envelope decoupling is primarily motivated by the very slow rotational velocities for the majority of the $\mathrm{G}$ dwarfs at young age and by the apparent longer spindown timescales as one goes to lower masses (c.f. Stauffer, Hartmann \& Latham 1987). The bimodal rotational velocity distribution for the late $\mathrm{G}$ and $\mathrm{K}$ dwarfs of the Pleiades is possibly a by-product of all these mechanisms - core-envelope decoupling and disk-locking to encourage rapid spindown of the initially more slowly rotating stars, and saturation of the wind to allow the initially most rapidly rotating stars to retain their angular momentum for a longer period of time. However, the possible existence of a bimodal rotational velocity distribution for $\mathrm{M}>0.5 \mathrm{M}_{\odot}$ stars in Orion and a more unimodal distribution at lower masses (Herbst, W., Bailer-Jones, \& Mundt 2001) may indicate that the rotational velocity distribution seen in the Pleiades was mostly present at age $\sim 1$ Myr. Definitively sorting out the true physical mechanisms which 

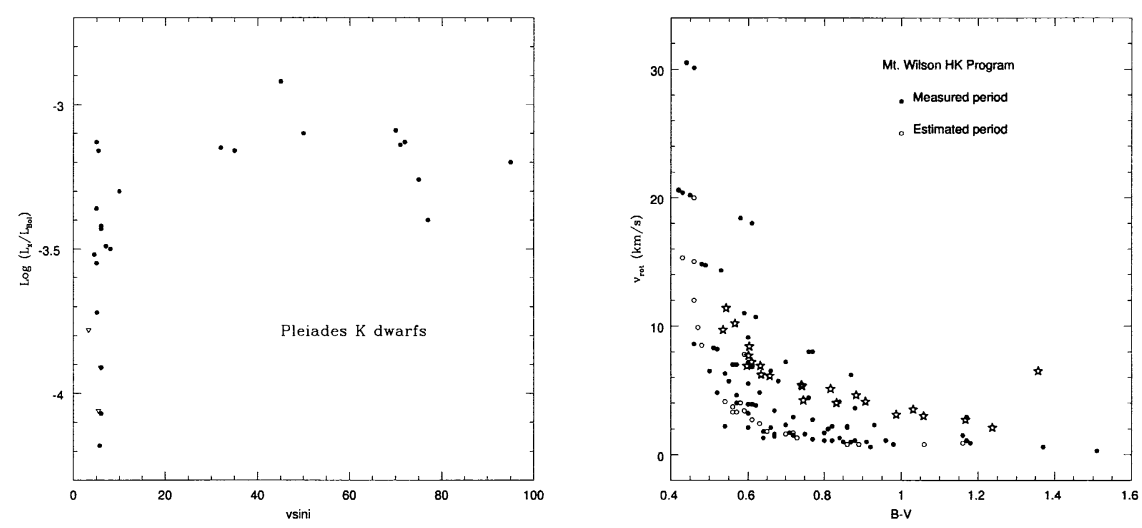

Figure 3. (a) Correlation between X-ray luminosity (normalized to bolometric luminosity) and $v \sin i$ for $\mathrm{K}$ dwarfs in the Pleiades. Similar relations are seen for Pleiades $\mathrm{M}$ dwarfs and for low mass stars in other open clusters. (b) Rotational velocities for the 112 low mass field stars from the Mt. Wilson CaHK monitoring program. Also plotted (as large five-pointed stars) are rotational velocities inferred for Hyades low mass stars from rotation periods derived by Radick et al. 1987).

lead to the rotational velocity distributions shown in Figure 1 remains a task for the future.

\section{Field Star Rotational Data}

Because the nearest open clusters are generally more than $100 \mathrm{pc}$ distant, it is possible to obtain much higher signal-to-noise photometry and spectroscopy of low mass field stars than for open cluster members of the same mass. Therefore, one might expect there to be a large and valuable database of rotational velocity data for low mass field stars. However, two factors conspire against that expectation. First, assuming a more or less constant star formation rate over the life of the disk, most field stars will be considerably older than the Hyades, and therefore their rotational velocities should be very small, generally providing only upper limits to $v \sin i$ determinations. Second, age estimates for low mass field stars are difficult to make and are significantly uncertain; utilizing these stars to determine evolutionary trends is correspondingly difficult. For these reasons, I will limit my discussion of field star rotation to two programs - the Mt. Wilson rotational periods and a spectroscopic survey of field $\mathrm{M}$ dwarf rotation. My goal is simply to determine if there is any evidence that the rotational velocity history for field stars differs from that for open cluster members. In my opinion, that is a priori improbable, given that most field stars are likely to have been members of open clusters that have become gravitationally unbound over time.

The Mt. Wilson group has been obtaining precision, narrow-band photometry of the flux in the CaII HK resonance lines for a sample of about 110 low 
mass field stars for more than three decades (Donahue, Saar \& Baliunas 1996; Baliunas, Sokoloff \& Soon 1996). For the majority of those stars, they have been able to derive rotational periods from one or more of their annual datasets. Where they have not been able to directly determine a rotation period, they believe they can accurately estimate the rotation period from the mean CaHK flux. Figure $3 \mathrm{~b}$ shows the inferred distribution of rotational velocities for this sample of late F to early M dwarfs. Superimposed on that figure are rotational velocities inferred from the rotation periods determined for Hyades low mass dwarfs by Radick et al. (1987). The shape of the field star distribution is qualitatively the same as for the Hyades, and the number of field dwarfs rotating faster than Hyades stars of the same B-V seems plausible given the age of the Hyades and an assumed range of ages up to $>5$ Gyr for the field stars. The range in rotation at a given mass in the Hyades is obviously very small, indicating that the main sequence angular momentum loss process must indeed spin down the rapid rotators more aggressively than the slow rotators (rotation period data shows this much more clearly than spectroscopic rotational velocities due to the "noise" added by $\sin i$ and the lower intrinsic precision of the $v \sin i$ data).

The best extant study of the rotational velocity distribution of field $M$ dwarfs is that by Delfosse et al. (1998). Those authors obtained high signal-tonoise, high resolution spectra of a sample of 118 field $M$ dwarfs. Their Figure 3 indicates that while most late $M$ dwarfs have very small rotational velocities, a significant fraction of the young $M$ dwarfs have rotational velocities ranging up to $30 \mathrm{~km} \mathrm{~s}^{-1}$. Old disk $\mathrm{M}$ dwarfs clearly have rotational velocities less than those for the young $\mathrm{M}$ dwarfs, but even some of these stars (particularly the very latest with $\mathrm{R}-\mathrm{I}>2.0$ ) have rotational velocities of order $10 \mathrm{~km} \mathrm{~s}^{-1}$. The field $\mathrm{M}$ dwarf data therefore corroborate the open cluster results, indicating that the spindown time for very low mass stars can be a significant fraction of the age of the galactic disk. Delfosse et al. also demonstrated that these field $M$ dwarfs show the same correlation between rotation and coronal activity as is found for the low mass open cluster stars, with an apparent critical velocity for saturation of order only a few $\mathrm{km} \mathrm{s}^{-1}$.

\section{Other Empirical Evidence Related to Rotational Evolution of Low Mass Stars}

It would seem at least plausible that the angular momentum loss rate from winds might be a function of stellar metallicity for low mass stars (e.g. via the depth of the convective envelope at a given mass - Jeffries et al. 1997). I have tacitly assumed that this effect, if present, is small, because I have used the Hyades rotational velocity distribution to compare to other clusters, despite the fact that the Hyades metallicity is significantly greater than solar $([\mathrm{Fe} / \mathrm{H}] \sim 0.14$ in the $\log$ relative to the Sun). A strong argument in favor of my view is provided by comparison of rotation periods for low mass stars in the Coma cluster to the Hyades rotational period distribution, because Coma is the same age as the Hyades but its metallicity is less than solar $([\mathrm{Fe} / \mathrm{H}] \sim-0.065)$. Figure 4a provides a comparison between the inferred Coma cluster rotational velocities (Radick, Skiff \& Lockwood 1990) and the equivalent data for the Hyades (Radick et al. 

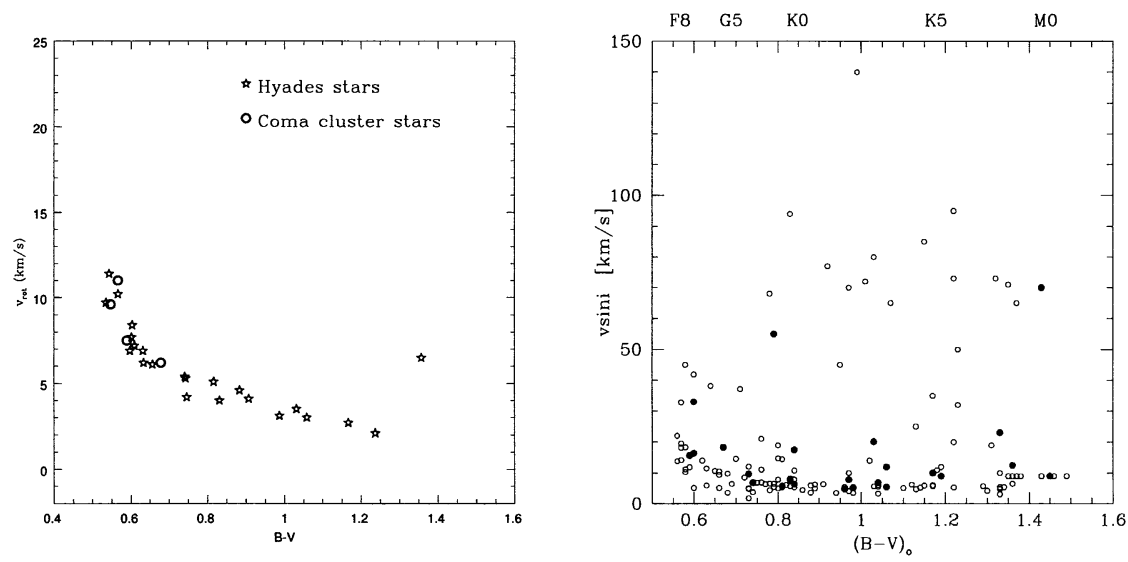

Figure 4. (a) Rotational velocity distribution for four solar mass stars in the slightly metal-poor Coma cluster (shown as large open circles), compared to the Hyades rotational velocites from Radick et al. 1987). (b) Rotational velocities of low mass Pleiades stars, with stars identified as binaries shown as filled dots and stars believed to be single shown as open circles. The two distributions do not appear to differ in any significant way.

1987). The figure shows that the Coma and Hyades stars of the same B-V have indistinguishable rotational velocities. This does indicate that at a given mass there will be some difference in rotation, but all of the figures I have shown are essentially as a function of color and therefore my assumption that the Hyades stars can be taken as "normal" for their age in a rotational sense appears justified.

Another physical parameter which one might think should significantly affect stellar rotation rate is binarity. The amount of angular momentum locked up in the orbital motion of two low mass stars will typically be greater than the inferred angular momentum present in even the most rapidly rotating, young low mass star. A plausible correlation therefore might be to expect low mass binary stars to be slowly rotating. Another mechanism, however, would predict an opposite correlation. The presence of a binary companion would be expected to limit the growth or truncate circumstellar disks during PMS evolution - and therefore one might expect that binary stars might have less long-lived circumstellar disks, hence be "disk-locked" for a shorter time, and hence arrive on the main sequence more rapidly rotating, on average, than single stars. However, the observational evidence suggests no obvious correlation between rotation and binarity. Figure $4 \mathrm{~b}$ illustrates this for the Pleiades, from a paper by Bouvier, Rigaut \& Nadeau (1997).

Finally, predictions have been made that one should see a change in the rotational velocity distribution at the mass below which main sequence stars are fully convective, or approximately at $0.3 \mathrm{M}_{\odot}$. This would result if, for example, the dominant dynamo mechanism above and below that mass were different. However, again, the data do not seem to show any change in the rotational velocity distributions for the open cluster stars (see Figure 1) at that 
mass. Similarly, Delfosse et al. did not see any evidence for a change in the distribution of rotational velocities for field $M$ dwarfs near $0.3 \mathrm{M}_{\odot}$.

\section{Conclusions}

At the time of the last international meeting on stellar rotation (Slettebak 1970), essentially nothing was known about the rotational velocities of stars less massive than the Sun. Modern technology has now allowed us to determine rotational velocities for hundreds of low mass stars in open clusters and to extend the empirical knowledge of stellar rotation to below the sub-stellar boundary. However, even with those data, the theoretical models remain very much underconstrained. Only one cluster (the Pleiades) has well-sampled rotational data over the entire 0.1 to $1.0 \mathrm{M}_{\odot}$ mass range. We have the means (multi-object echelle spectrographs on $8 \mathrm{~m}$ class telescopes for $v \sin i$ 's or large format, mosaiced CCD cameras for rotational periods) to obtain similar rotational data for many open clusters spanning a range of ages. Doing so should be a high priority to accomplish before the next stellar rotation meeting, which hopefully will occur prior to 2030 .

\section{References}

Baliunas, S., Sokoloff, D., \& Soon, W. 1996, ApJ 457, L99.

Barnes, S., Sofia, S., \& Pinsonneault, M. 2001, ApJ 548, 1071.

Bouvier, J., Rigaut, F, \& Nadeau, D. 1997, A\&A 323, 139.

Delfosse, X., Forveille, T., Perrier, C., \& Mayor, M. 1998, A\&A 331, 581.

Donahue, R., Saar, S., \& Baliunas, S. 1996, ApJ 466, 384.

Herbst, W., Bailer-Jones, C., \& Mundt, R. 2001, ApJ 554, L197.

Jeffries, R.D., Thurston, M., \& Pye, J. MNRAS 287, 350.

Jones, B.F., Fischer, D., \& Stauffer, J. 1996, AJ 112, 1562.

Kraft, R.P. 1967, ApJ 150, 551.

Mathieu, R. 2002, this conference.

Oppenheimer, B., Basri, G., Nakajima, T., \& Kulkarni, S. 1997, AJ 113, 296.

Paulson, D., \& Cochran, W. 2002, priv. comm.

Queloz, D, Allain, S., Mermilliod, J.-C., Bouvier, J., \& Mayor, M. 1998, A\&A 335, 183.

Radick, R., Skiff, B., \& Lockwood, G. 1990, ApJ 353, 524.

Radick, R., Thompson, D., Lockwood, G., Duncan, D., \& Baggett, W. 1987, ApJ 321, 459.

Schatzman, E. 1962, Ann. d'Ap, 25, 18.

Sills, A., Pinsonneault, M., \& Terndrup, D. 2000, ApJ 534, 335.

Slettebak, A. 1970, IAU Colloquium 4, "Rotation, Stellar Interiors, Stellar Evolution and Stellar Atmospheres (Dordrecht: Reidel).

Soderblom, D., Jones, B.F., \& Fischer, D. 2001, ApJ 563, 334.

Stauffer, J., Hartmann, L., \& Latham, D. 1987, ApJ 320, L51.

Terndrup, D., Stauffer, J., Pinsonneault, M., et al. 2000, AJ 119, 1303.

Terndrup, D., Pinsonneault, M., Jeffries, R.D., Ford, A., Stauffer, J., and Sills, A. 2002, ApJ 576, 950. 